\title{
Transient high-level expression of ß-galactosidase after transfection of fibroblasts from GM1 gangliosidosis patients with plasmid DNA
}

\author{
R.C. Balestrin ${ }^{1,4}$, G. Baldo 2,4 , M.B. Vieira ${ }^{2,3}$, R. Sano ${ }^{2}$, J.C. Coelho2,3, R. Giugliani1,2,3,4 \\ and U. Matte ${ }^{4}$ \\ ${ }^{1}$ Programa de Pós-Graduação em Genética e Biologia Molecular, ${ }^{2}$ Departamento de Bioquímica, \\ Universidade Federal do Rio Grande do Sul, Porto Alegre, RS, Brasil \\ ${ }^{3}$ Serviço de Genética Médica, ${ }^{4}$ Centro de Terapia Gênica, Hospital de Clínicas de Porto Alegre, \\ Porto Alegre, RS, Brasil
}

Correspondence to: U. Matte, Centro de Terapia Gênica, HCPA, Rua Ramiro Barcelos, 2350, 90035-903 Porto Alegre, RS, Brasil

Fax: +55-51-2101-8010. E-mail: umatte@hcpa.ufrgs.br

GM1 gangliosidosis is an autosomal recessive disorder caused by the deficiency of lysosomal acid hydrolase $ß$-galactosidase (ß-Gal). It is one of the most frequent lysosomal storage disorders in Brazil, with an estimated frequency of 1:17,000. The enzyme is secreted and can be captured by deficient cells and targeted to the lysosomes. There is no effective treatment for GM1 gangliosidosis. To determine the efficiency of an expression vector for correcting the genetic defect of GM1 gangliosidosis, we tested transfer of the ß-Gal gene (Glb1) to fibroblasts in culture using liposomes. ß-Gal cDNA was cloned into the expression vectors pSCTOP and pREP9. Transfection was performed using $4 \mu \mathrm{L}$ lipofectamine 2000 and 1.5-2.0 $\mu$ g DNA. Cells ( $2 \times 105 /$ well) were harvested $24 \mathrm{~h}, 48 \mathrm{~h}$, and 7 days after transfection. Enzyme specific activity was measured in cell lysate and supernatant by fluorometric assay. Twenty-four hours after transfection, treated cells showed a higher enzyme specific activity (pREP9-ßGal: $621.5 \pm 323.0$, pSCTOP-ß-Gal: $714.5 \pm 349.5$, pREP9-ß-Gal + pSCTOP-ß-Gal: $1859.0 \pm 182.4$, and pREP9-ß-Gal + pTRACER: $979.5 \pm 254.9 \mathrm{nmol} \cdot \mathrm{h}^{-1} \cdot \mathrm{mg}^{-1}$ protein) compared to untreated cells $\left(18.0 \pm 3.1\right.$ for cell and $32.2 \pm 22.2 \mathrm{nmol} \cdot \mathrm{h}^{-1} \cdot \mathrm{mg}^{-1}$ protein for supernatant). However, cells maintained in culture for 7 days showed values similar to those of untreated patients. In the present study, we were able to transfect primary patients' skin fibroblasts in culture using a non-viral vector which overexpresses the $ß-G$ al gene for $24 \mathrm{~h}$. This is the first attempt to correct fibroblasts from patients with GM1 gangliosidosis by gene therapy using a non-viral vector.

Key words: GM1 gangliosidosis; ß-galactosidase deficiency; Gene therapy; Lysosomal storage disorder; Lipofectamine

Research supported by grants from PROABI (FAPERGS), Program for Centers of Excellence (PRONEX-MCT), CAPES, and FIPE-HCPA.

Received March 9, 2007. Accepted February 18, 2008

\section{Introduction}

GM1 gangliosidosis (OMIM \#230500) is an autosomal recessive lysosomal storage disorder (LSD) caused by deficiency of the acid hydrolase ß-galactosidase (GLB1; EC 3.2.1.23). The disorder leads to progressive neural and visceral accumulations of GM1 ganglioside, its asialo derivative GA1, and other minor glycolipids and glycopeptides (1). GM1 gangliosidosis is one of the most common LSD in Southern Brazil, with an estimated incidence of $1: 17,000$ live births (2).

GM1 gangliosidosis is characterized by generalized 
involvement of the central nervous system (CNS), progressive neurodegeneration, and early death (3). Three clinical phenotypes can be distinguished, classified by age of onset: infantile, late infantile/juvenile, and adult. Infantile GM1 gangliosidosis, the severe form of the disease, is characterized by facial and skeletal abnormalities and neurological deterioration starting at about 6 months of age. Most patients die within the first 2 years of age (3).

To date, there is no effective treatment for GM1 gangliosidosis, mainly due to the neurological involvement caused by the disease. As in other LSD with CNS involvement, the enzyme is unable to cross the blood brain barrier. A strategy to overcome this hurdle could be the direct gene transfer to the CNS or the introduction of genetically modified cells into the brain (4). Other strategies attempted for GM1 gangliosidosis include the use of adenoviral vectors injected systemically during the neonatal period (5) and the genetic modification of bone marrow stem cells (6). Both studies were conducted using the murine model of GM1 gangliosidosis and showed promising results, although the feasibility of translating such strategies to clinical trials remains to be assessed.

The aim of the present study was to determine whether the biochemical defect in human GM1 gangliosidosis fibroblasts could be corrected by using non-viral gene delivery. In addition, the best expression vector for $ß$-galactosidase (ß-Gal) overexpression was also identified.

\section{Material and Methods}

\section{Cell culture}

Human fibroblasts were obtained from skin biopsies from a healthy individual and from a patient with GM1 gangliosidosis. The primary culture of fibroblasts was maintained in Dulbecco's modified Eagle's medium (DMEM) (Invitrogen, USA) supplemented with $10 \%$ fetal calf serum (Cultilab, Brazil) at $37^{\circ} \mathrm{C}$ in the presence of $5 \% \mathrm{CO}_{2}$. The day before transfection, cells were plated onto 24-well plates at a density of $2 \times 10^{5}$ cells/well to reach $90-95 \%$ confluence ( $\mathrm{N}=4$ or 5 plates/group).

\section{Plasmid construction}

The ß-Gal cDNA (kindly provided by Dr. Alessandra D'Azzo, Memphis, TN, USA) was cloned into two expression plasmids: pSCTOP and pREP9 (kindly provided by Dr. Nance Nardi, Federal University of Rio Grande do Sul, Brazil). The $ß-G a l$ cDNA in the pSCTOP vector was under the control of the cytomegalovirus (CMV) promoter. The pREP9 vector is an Epstein-barr virus (EBV)-based vector containing the Rous sarcoma virus (RSV) promoter. The pREP9-ß-Gal was transfected alone or co-transfected with pSCTOP ß-Gal and pTRACER (Invitrogen). This latter vector contains a green fluorescent protein marker gene and no $ß-G a l$ cDNA. In addition, pTRACER alone and pREP9 without the $ß-G a l$ cDNA were also transfected.

All plasmids were grown and isolated from Escherichia coli (DH5- $\alpha$ cells; Invitrogen) by standard procedures (7) and purified using the Concert Midiprep Kit (Concert High Purity Purification Systems, Gibco, USA).

\section{Transfection procedure}

Transfections were performed using the lipofectamine 2000 reagent (Invitrogen) in serum-free DMEM for $24 \mathrm{~h}$ according to manufacturer instructions. The amounts of lipofectamine 2000 used were $4 \mu \mathrm{L}$ for $1.5 \mu \mathrm{g}$ plasmid $(2 \mu \mathrm{g}$ for co-transfection) in $95 \%$ confluent cells plated onto 24 well plates (approximately $2 \times 10^{5}$ cells/well).

\section{ß-Gal-specific activity assay}

Cells were assayed at different times ( 24 h, 48 h, and 7 days) after transfection. No selective antibiotics were added. Enzyme assays for ß-Gal specific activity were performed in cell lysate (obtained by sonication) by the fluorimetric method of Suzuki (8), using 4-methylumbelliferyl ß-Gal as substrate (excitation $365 \mathrm{~nm}$ and emission $450 \mathrm{~nm}$ ). Protein content was previously determined by the method of Lowry et al. (9). The enzyme assay was performed in treated and untreated fibroblasts from GM1 gangliosidosis patients and in fibroblasts from a healthy individual, as control.

\section{Cell viability assay}

Viability was assessed by the tetrazolium 3-(4,5-dimethylthiazol-2-yl)-2,5 phenyltetrazolium bromide test (MTT; Sigma Chemical, USA) using $10^{4}$ cells per well in 96-well plates in GM1 fibroblasts transfected with all the vectors used in this study ( $N=4$ independent tests/group). Briefly, the culture medium was removed and MTT (0.5 $\mathrm{mg} / \mathrm{mL}$ in DMEM) was added to each well and incubated for $4 \mathrm{~h}$ at $37^{\circ} \mathrm{C}$. After lysing cells with $100 \mu \mathrm{L}$ DMSO, viability was assessed by measuring absorbance at 570 $\mathrm{nm}$ using a microplate reader (BioRad, USA). The color intensity observed in each well was correlated to the number of viable cells, considering absorbance in nontransfected cells as $100 \%$ viability.

\section{Semi-quantitative PCR}

In order to quantify the efficiency of gene transfer, semi-quantitative PCR was applied to DNA extracted from cells $24 \mathrm{~h}$ after transfection. The standard curve was based on increasing concentrations of pREP-ß-Gal cDNA ranging from 50 to $200 \mathrm{ng}$. The intensity of bands was quantified using the UVIDoc Software and plotted against the 
curve. PCR conditions were as follows: primers (Exon6A 5' ggcagctactttgcctgtg 3' and Exon6B 5' ggctcacacttcctctggc $3^{\prime}$ ) were used at $20 \mathrm{pmol} / \mu \mathrm{L}$ with an annealing temperature of $57^{\circ} \mathrm{C}$ for 20 cycles. Reactions were prepared using $\mathrm{KCl}$ buffer, $0.2 \mathrm{mM}$ dNTP, $1.5 \mathrm{mM} \mathrm{MgCl}_{2}$, and $1 \mathrm{U}$ Taq Polymerase. All reagents were purchased from Invitrogen.

\section{Statistical analysis}

Differences in enzyme specific activity and cell viability among groups were determined using the Statistical Package for Social Sciences. One-way ANOVA and the post hoc Tukey test were used for statistical analysis, with $\mathrm{P}<$ 0.05 being considered statistically significant.

\section{Ethical issues}

This study was approved by the Ethics Committee of Hospital de Clínicas de Porto Alegre (study No. 02-084). As the study was performed on unidentified samples, the Ethics Committee waived the need for informed consent.

\section{Results}

To evaluate expression of the ß-Gal gene, human fibroblasts from GM1 patients were transfected with pSCTOP-ß-Gal alone, pREP9-ß-Gal alone and co-transfected with pREP9-ß-Gal and pSCTOP-ß-Gal or pREP9$ß-G a l$ and $p$ TRACER using lipofectamine 2000. Transfections with pREP9 alone and pTRACER alone were also performed.

The optimal conditions for transfection, previously determined using healthy fibroblasts, were a density of 2 x $10^{5}$ cells per well and a 1:2 ratio for DNA/lipofectamine 2000 (data not shown). Enzyme specific activity was measured in the cell lysate and supernatant by a fluorometric assay at $24 \mathrm{~h}$, $48 \mathrm{~h}$, and 7 days after transfection. Enzyme specific activity (mean \pm SD) was also measured in cells and supernatant from untreated fibroblasts from GM1 gangliosidosis patients (18.0 \pm $3.1,32.2 \pm 22.2 \mathrm{nmol} \cdot \mathrm{h}^{-1} \cdot \mathrm{mg}^{-1}$ protein) and in healthy control fibroblasts and supernatant $(893.7 \pm 311.9,529.0 \pm$ $144.5 \mathrm{nmol} \cdot \mathrm{h}^{-1} \cdot \mathrm{mg}^{-1}$ protein) for comparison. This specific activity was maintained throughout the 7 days of the study.

The transfection of vectors without ß-Gal cDNA did not cause any increase ß-galactosidase. in enzyme specific activity. Transfection with pTRACER produced a mean specific activity of $17.5 \pm 9.3,12.2 \pm 7.5$, and $13.8 \pm 10.8 \mathrm{nmol} \cdot \mathrm{h}^{-1} \cdot \mathrm{mg}^{-1}$ protein for cell lysate and $21.3 \pm 5.9,24.3 \pm 11.7$, and $22.9 \pm 7.6 \mathrm{nmol} \cdot \mathrm{h}^{-1} \cdot \mathrm{mg}^{-1}$ protein for supernatant after $24 \mathrm{~h}, 48 \mathrm{~h}$, and 7 days, respectively. Similarly, pREP9 transfection produced values of $15.9 \pm 5.0,23.2 \pm 8.6$, and $20.0 \pm 7.8$ in fibroblasts and $25.3 \pm 11.5,16.3 \pm 6.5$, and $20.45 \pm 2.7$ in the supernatant for the same times post-transfection.

The enzyme specific activities of control and treated cells are shown in Table 1 as the mean value for four independent determinations $(\mathrm{N}=4)$, except for the cotransfection of pREP9-ß-Gal and pSCTOP-ß-Gal ( $N=5)$. Single transfection of pREP9-ß-Gal and pSCTOP-ß-Gal showed enzyme specific activity levels close to those of healthy cells. As shown in Table 1, the enzyme specific activity of treated GM1 fibroblasts was considerably higher 24 and $48 \mathrm{~h}$ after transfection when compared to untreated patients $(P<0.05)$.

Co-transfection of pREP9-ß-Gal with other plasmids (pREP9-ß-Gal + pSCTOP-ß-Gal or pREP9-ß-Gal + pTRACER) raised the specific activity to levels above those observed with pREP9-ß-Gal alone. The combination of vectors pREP9-ß-Gal and pSCTOP-ß-Gal showed the highest values for enzyme specific activity, both at 24 and $48 \mathrm{~h}(\mathrm{P}<0.05)$. Co-transfection with pREP9-ß-Gal + pTRACER was higher than transfection with pREP9-ß-Gal

Table 1. Enzyme specific activity in cell lysate and supernatant of GM1 fibroblasts after transfection with pREP9-ß-Gal $(N=4)$, pSCTOP- $B-G a l(N=4)$, and co-transfection with pREP9-ß-Gal + pTRACER $(N=4)$ and pREP9-ß-Gal + pSCTOP-ß-Gal $(N=5)$.

\begin{tabular}{|c|c|c|c|}
\hline & $24 \mathrm{~h}$ & $48 \mathrm{~h}$ & 7 days \\
\hline \multicolumn{4}{|l|}{ pREP9-ß-Gal } \\
\hline Cell lysate & $621.5 \pm 323.0^{a}$ & $619.1 \pm 291.2^{\mathrm{a}}$ & $173.3 \pm 88.8^{b}$ \\
\hline Supernatant & $629.5 \pm 82.3^{a}$ & $448.8 \pm 228.2^{\mathrm{a}}$ & $154.3 \pm 29.9^{b}$ \\
\hline \multicolumn{4}{|l|}{ pSCTOP-ß-Gal } \\
\hline Cell lysate & $714.5 \pm 349.5^{a}$ & $660.3 \pm 307.2^{\mathrm{a}}$ & $100.4 \pm 48.1^{b}$ \\
\hline Supernatant & $688.7 \pm 361.6^{a}$ & $577.6 \pm 349.5^{a}$ & $114.1 \pm 35.4^{b}$ \\
\hline \multicolumn{4}{|c|}{ pREP9-ß-Gal + pTRACER } \\
\hline Cell lysate & $979.5 \pm 254.9^{a}$ & $698.8 \pm 365.5^{a}$ & $114.1 \pm 22.9^{b}$ \\
\hline Supernatant & $579.4 \pm 190.1^{a}$ & $519.8 \pm 90.0^{a}$ & $112.3 \pm 64.3^{\mathrm{b}}$ \\
\hline \multicolumn{4}{|c|}{ pREP9-ß-Gal + pSCTOP-ß-Gal } \\
\hline Cell lysate & $1859.0 \pm 182.4^{\mathrm{a}, \mathrm{b}, \mathrm{c}}$ & $1608.7 \pm 223.1^{a, b, c}$ & $183.9 \pm 77.1^{b}$ \\
\hline Supernatant & $1467.5 \pm 208.5^{a, b, c}$ & $1148.3 \pm 204.6^{a, b, c}$ & $217.2 \pm 94.0^{\mathrm{a}, \mathrm{b}}$ \\
\hline
\end{tabular}

Enzyme specific activity is reported as $\mathrm{nmol} \cdot \mathrm{h}^{-1} \cdot \mathrm{mg}^{-1}$ protein. Data are reported as mean \pm SD. Mean reference values $(N=4)$ for healthy individuals: $893.7 \pm 311.9$ for cell lysate and $529.0 \pm 144.5$ for supernatant, and mean values for affected individuals: $18.0 \pm 3.1$ for cell lysate and $32.2 \pm 22.2$ for supernatant.

ap $<0.05$ compared to affected individuals; bP $<0.05$ compared to normal individuals;

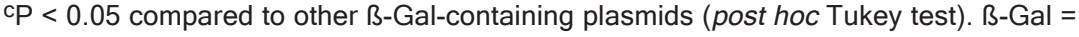


alone, although this difference was not statistically significant.

However, all groups transfected with ß-Gal-containing plasmids showed a marked reduction in enzyme levels after 7 days when compared to 24 and 48 h. Enzyme specific activity after 7 days of culture did not differ significantly from that of untreated GM1 fibroblasts ( $P>0.05$ ), except for the supernatant from pREP9-ß-Gal + pSCTOPß-Gal co-transfected cells.

Although the MTT test at 7 days showed that the percent of viable cells decreased in the pREP9-ß-Gal and in the pREP9-ß-Gal + pSCTOP-ß-Gal groups, cell viability in all transfections and at all times analyzed was higher than $75 \%$, indicating that loss of enzyme expression at 7 days did not occur due to cell death induced by plasmid toxicity. The only group that showed a marked reduction in cell viability after $24 \mathrm{~h}, 48 \mathrm{~h}$, and 7 days was the group with cells treated with lipofectamine alone (Figure 1), whose values were reduced to less than $50 \%$ after one week. The MTT test for transfections with pTRACER and pREP9 without $ß-G a l$ cDNA showed that viability was similar to that of the other groups (data not shown) and did not differ from that of non-transfected cells.

Differences in transfection efficiency were observed by

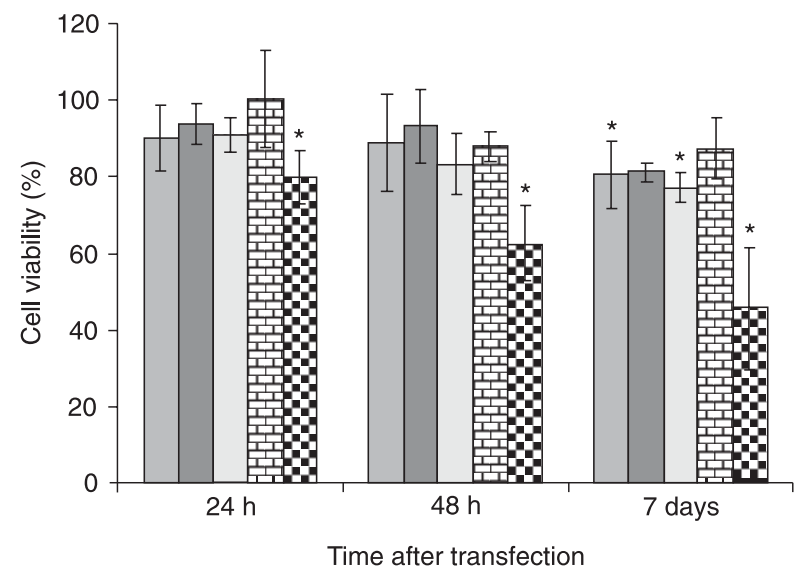

$\begin{array}{ll}\square \text { pREP9-ß-Gal } & \text { 回 pREP9-ß-Gal + pTRACER } \\ \square \text { pSCTOP-ß-Gal } & \text { 四 Lipofectamine } \\ \square \text { pREP9-ß-Gal + pSCTOP-ß-Gal } & \end{array}$

Figure 1. MTT assay for evaluation of cell viability after transfec-

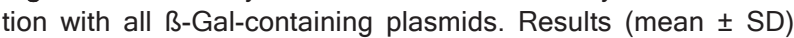
were compared to those obtained for untreated cells and are expressed as percent of viable cells at the indicated times $(\mathrm{N}=4$ plates/group). ${ }^{*} \mathrm{P}<0.05$ compared to the untreated group (post hoc Tukey test). MTT = tetrazolium 3-(4,5-dimethylthiazol-2-yl)2,5 phenyltetrazolium bromide; $ß$-Gal = $ß$-galactosidase.
PCR analysis comparing band intensity to a serial dilution of $ß-G a l$ cDNA. When the absorbances of the PCR products were plotted against the values obtained from the serial dilution $p R E P 9-ß-G a l$ and $p S C T O P-ß-G a l$ presented values of about $75 \mathrm{ng}$ whereas pREP9-ß-Gal + pTRACER and pREP9-ß-Gal + pSCTOP-ß-Gal presented values over $200 \mathrm{ng}$ (data not shown).

\section{Discussion}

Gene transfer to cells inside the CNS may represent a powerful tool to treat a large number of diseases, including neurological disorders and lysosomal diseases with CNS involvement (4). Research in this area has been mainly based on the use of viral vectors due to their high gene transfer efficiency. Nevertheless, important safety limitations have been associated with viral vectors, including the risk of immunogenicity, cytotoxicity, and tumorigenicity (10-13). For gene therapy treatment to be effective, CNS symptoms should be detected as soon as possible, to avoid irreversible damage.

The first study on gene therapy for GM1 gangliosidosis was published in 2000 by Sena-Esteves et al. (14), showing that transduction of human fibroblasts with retroviral vectors encoding the human acid $ß-G a l$ cDNA leads to complete correction of the enzyme deficiency. The synthesized enzyme was correctly processed and targeted to the lysosomes in transduced cells. However, cross-correction experiments showed that the human enzyme is taken up with low efficiency. The authors suggested that secreted levels of $ß-G a l$ in humans may be lower than for other lysosomal enzymes.

In the present study, we investigated whether the biochemical defect in human GM1 fibroblasts could be corrected by non-viral gene delivery. Cationic liposomes are the most widely used non-viral vectors, improving transfection efficiency as well as having adequate features for in vivo use in clinical and preclinical trials $(15,16)$.

Our results suggest that gene therapy for GM1 gangliosidosis is feasible since enzyme specific activity in treated fibroblasts reached values almost 30 -fold the levels obtained in single transfections and a 100-fold increase with co-transfection after $24 \mathrm{~h}$, when compared to untreated GM1 cells. The highest level of enzyme specific activity was achieved with simultaneous transfection of vectors pREP9-ß-Gal + pSCTOP-ß-Gal.

The pSCTOP-ß-Gal plasmid used, which showed high levels of expression, is under the control of the CMV promoter. The CMV promoter, thought to be maximally active under basal conditions, has been used for many gene transfer experiments. However, more recently it has 
been shown to be regulated by many stimuli, such as $\alpha$ interferon, PMA/forskolin, and methyl-methanesulfonate in different cell types (17). It is inactivated after in vivo gene transfer into the mouse liver and muscle. In vitro studies on cell line tumors revealed that a decrease in transgene mRNA levels is associated with extensive methylation (1820). In our study, silencing was not observed because the experiments were conducted in vitro for a short period of time.

The pREP9-ß-Gal vector is under the control of the RSV promoter. As an EBV-based vector, it has EBV ori-P as an origin of replication and the EBNA-1 gene for the EBV nuclear antigen, plus the neomycin-resistance gene $\left(\right.$ neo $\left.^{\circledR}\right)$. These elements may be useful for in vitro selection in long-term experiments $(21,22)$. The RSV promoter has also been extensively used for gene transfer, especially to the myocardium. This promoter, however, has been less well characterized than the CMV promoter (17). The pREP9ß-Gal controlled by the RSV promoter showed similar enzyme specific activity levels when compared to pSCTOPß-Gal.

Co-transfection of two vectors simultaneously led to increased levels of enzyme specific activity. The combination of pREP9-ß-Gal + pSCTOP-ß-Gal increased enzyme specific activity significantly when compared to all other groups (100X above untreated cells at $24 \mathrm{~h}$ ). Co-transfection of pREP9-ß-Gal + pTRACER led to values similar to those of normal individuals and increased enzyme specific activity over pREP9-ß-Gal alone at $24 \mathrm{~h}$, although the difference was not statistically significant.

Other studies have shown that co-transfection of multiple therapeutic genes is possible and can be more effective than monotherapy. Usually this is explained by the synergistic effect of transfected genes, as shown by Daddi et al. (23), Jeschke and Klein (24), and Suda et al. (25). In 2002, Daddi et al. (23) evaluated the effects of interleukin 10 (IL-10) and transforming growth factor $\aleph_{1}$ (TGF- $ß_{1}$ ) and their anti-inflammatory role in lung transplant ischemia- reperfusion injury and demonstrated that the co-transfection of TGF- $ß_{1}+$ IL-10 has a synergistic effect in ameliorating lung perfusion injury after prolonged ischemia. In 2005, the same group (25) showed that this synergistic effect leads to suppression of IL-2, thus reducing acute lung graft rejection. In 2004, Jeschke and Klein (24) demonstrated that the combined use of a mesenchymal (IGF-I) and epithelial (KGF) growth factor has an additive effect on the physiological and biological response, enhancing dermal and epidermal regeneration. These investigators proposed that multiple gene transfer may represent a possible clinical approach.

The decrease in $ß-$-Gal levels after 7 days does not seem to be due to cell death, since results obtained from the MTT assay showed that cell viability was always higher than $75 \%$, except when lipofectamine was added without any plasmid. This toxicity is due to the positive charge of lipofectamine, which in all other cases was balanced by the plasmid DNA-negative phosphate groups. The reduction in cell viability found for pREP9-ß-Gal and for the cotransfection of pREP9-ß-Gal + pSCTOP-ß-Gal 7 days after transfection, although statistically significant, does not, by itself, explain the loss of $ß-$ Gal specific activity observed after this period, since this was a common feature to all other groups. It is more likely that these findings were due to loss of the plasmid, since no selection system was used.

The increase in enzyme specific activity shown here demonstrates that it is possible to transfect primary fibroblasts in culture using a non-viral vector. In addition, we were able to overexpress the $ß-G a l$ gene for a short period of time, opening the possibility for cross-correction studies.

We report here the first attempt to correct fibroblasts from patients with GM1 gangliosidosis by gene therapy using a non-viral vector, leading to the use of a stable nonintegrative vector and to the potential transfer of modified cells overexpressing the $ß-$ Gal gene in an animal model of GM1 gangliosidosis.

\section{References}

1. Matsuda J, Suzuki O, Oshima A, Yamamoto Y, Noguchi A, Takimoto K, et al. Chemical chaperone therapy for brain pathology in G(M1)-gangliosidosis. Proc Natl Acad Sci U S A 2003; 100: 15912-15917.

2. Severini $M H$, Silva CD, Sopelsa A, Coelho JC, Giugliani R. High frequency of type $1 \mathrm{GM} 1$ gangliosidosis in southern Brazil. Clin Genet 1999; 56: 168-169.

3. Suzuki Y, Sakaraba H, Oshima A. ß-Galactosidase deficiency (ß-galactosidase): GM1 gangliosidosis and Morquio

B disease. In: Scriver CR, Beaudet AI, Sly WS, Valle DV (Editors), The metabolic and molecular basis of inherited disease. 8th edn. New York: McGraw Hill; 2001. p 37753809.

4. Schlachetzki F, Zhang Y, Boado RJ, Pardridge WM. Gene therapy of the brain: the trans-vascular approach. Neurology 2004; 62: 1275-1281.

5. Takaura N, Yagi T, Maeda M, Nanba E, Oshima A, Suzuki $\mathrm{Y}$, et al. Attenuation of ganglioside GM1 accumulation in the 
brain of GM1 gangliosidosis mice by neonatal intravenous gene transfer. Gene Ther 2003; 10: 1487-1493.

6. Sano R, Tessitore A, Ingrassia A, d'Azzo A. Chemokineinduced recruitment of genetically modified bone marrow cells into the CNS of GM1-gangliosidosis mice corrects neuronal pathology. Blood 2005; 106: 2259-2268.

7. Sambrook J, Fritsch EF, Maniatis T. Molecular cloning: $A$ laboratory manual. 2nd edn. New York: Cold Spring Harbor Laboratory Press; 1989.

8. Suzuki K. Globoid cell leukodystrophy (Krabe disease) and GM1 gangliosidosis. In: Glew RH, Peters SP (Editors), Practical enzymology of the sphingolipidoses. New York: Alan R. Liss; 1977. p 101-136.

9. Lowry $\mathrm{OH}$, Rosebrough NJ, Farr AL, Randall RJ. Protein measurement with the Folin phenol reagent. J Biol Chem 1951; 193: 265-275.

10. Somia N, Verma IM. Gene therapy: trials and tribulations. Nat Rev Genet 2000; 1: 91-99.

11. Thomas M, Klibanov AM. Non-viral gene therapy: polycation-mediated DNA delivery. Appl Microbiol Biotechnol 2003; 62: 27-34.

12. Mikkers H, Berns A. Retroviral insertional mutagenesis: tagging cancer pathways. Adv Cancer Res 2003; 88: 53-99.

13. Hacein-Bey-Abina $S$, Von Kalle $C$, Schmidt M, Le Deist $F$, Wulffraat N, McIntyre E, et al. A serious adverse event after successful gene therapy for X-linked severe combined immunodeficiency. N Engl J Med 2003; 348: 255-256.

14. Sena-Esteves M, Camp SM, Alroy J, Breakefield XO, Kaye EM. Correction of acid beta-galactosidase deficiency in GM1 gangliosidosis human fibroblasts by retrovirus vector-mediated gene transfer: higher efficiency of release and crosscorrection by the murine enzyme. Hum Gene Ther 2000; 11: 715-727.

15. Armeanu S, Pelisek J, Krausz E, Fuchs A, Groth D, Curth R, et al. Optimization of nonviral gene transfer of vascular smooth muscle cells in vitro and in vivo. Mol Ther 2000; 1 : 366-375.

16. Johansson A, Moller C, Harper P. Correction of the biochemical defect in porphobilinogen deaminase deficient cells by non-viral gene delivery. Mol Cell Biochem 2003; 250: 65-71.

17. Maass A, Langer SJ, Oberdorf-Maass S, Bauer S, Neyses $\mathrm{L}$, Leinwand LA. Rational promoter selection for gene transfer into cardiac cells. J Mol Cell Cardiol 2003; 35: 823-831.

18. Teschendorf C, Warrington KH Jr, Siemann DW, Muzyczka $\mathrm{N}$. Comparison of the EF-1 alpha and the CMV promoter for engineering stable tumor cell lines using recombinant adeno-associated virus. Anticancer Res 2002; 22: 33253330.

19. Grassi G, Maccaroni P, Meyer R, Kaiser H, D'Ambrosio E, Pascale E, et al. Inhibitors of DNA methylation and histone deacetylation activate cytomegalovirus promoter-controlled reporter gene expression in human glioblastoma cell line U87. Carcinogenesis 2003; 24: 1625-1635.

20. Brooks AR, Harkins RN, Wang P, Qian HS, Liu P, Rubanyi GM. Transcriptional silencing is associated with extensive methylation of the CMV promoter following adenoviral gene delivery to muscle. J Gene Med 2004; 6: 395-404.

21. Yates JL, Warren N, Sugden B. Stable replication of plasmids derived from Epstein-Barr virus in various mammalian cells. Nature 1985; 313: 812-815.

22. Morozova OV, Maksimova TG, Kostenko EV. EBV-based plasmid DNA rearrangements after transfection of eukaryotic cells. Plasmid 2000; 43: 185-189.

23. Daddi N, Suda T, D'Ovidio F, Kanaan SA, Tagawa T, Grapperhaus K, et al. Recipient intramuscular cotransfection of naked plasmid transforming growth factor beta1 and interleukin 10 ameliorates lung graft ischemia-reperfusion injury. J Thorac Cardiovasc Surg 2002; 124: 259-269.

24. Jeschke MG, Klein D. Liposomal gene transfer of multiple genes is more effective than gene transfer of a single gene. Gene Ther 2004; 11: 847-855.

25. Suda T, Daddi N, Tagawa T, Kanaan SA, Kozower BD, Ritter $\mathrm{JH}$, et al. Recipient intramuscular cotransfection of transforming growth factor beta 1 and interleukin 10 ameliorates acute lung graft rejection. $J$ Thorac Cardiovasc Surg 2005; 129: 926-931. 